\title{
Tuberkulose als Indikator für die Besiedlung der Erde
}

Die Tuberkulose (TB) stammt aus Afrika und verbreitete sich vor rund 43000 Jahren über die ganze Welt - das hat die rekonstruierende Genanalyse aller gegenwärtig bekannten Tuberkulosestämme gezeigt. Die Analyse von I. Comas et al. ist dabei nicht nur medizinisch von Relevanz: Für Historiker werden frühgeschichtliche Migrationsströmungen erkennbar, da diese die Genvariationen beeinflussten. Nat Genet 2013; 45: 1176-1182

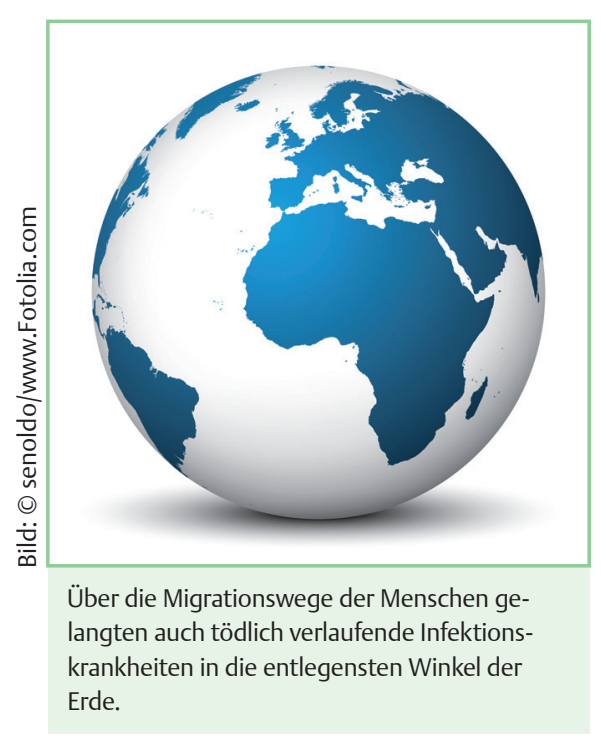

Die Tuberkulose (M. tuberculosis complex: MTBC) kommt in dieser Form nur bei Menschen vor. Sie gilt als eine der ältesten humanen Infektionskrankheiten. Mit der modernen Genanalyse ist es möglich, das Genom der Ursprungsstämme zu rekonstruieren. Aus der Diversifizierung der heutigen Stämme und unter Berücksichtigung der Geschwindigkeit, mit der genetische Veränderungen auftreten, sind solche historischen Rekonstruktionen möglich. Deshalb ist die Geschichte der TB auch ein Abbild der frühgeschichtlichen Migrationsbewegungen. Selbst prähistorische Phasen der Bevölkerungsverdichtung sind so indirekt nachweisbar.

\section{Der Urstamm der Tuberkulose $\nabla$}

Das internationale Forscherkonsortium um I. Comas analysierte in seiner genetischen Studie alle 259 weltweit verbreite- ten Tuberkulosestämme. Rund 34000 genetische Variationen wurden dabei erfasst. Deren Analyse zeigt, dass der Urstamm der TB etwa 73000 Jahre alt sein muss. Der Ursprung der Krankheit liegt in Afrika.

Bereits vor 63000 Jahren kam es zu ersten Wanderungsbewegungen, die die Tuberkulose bis zum Indischen Ozean trugen. Massive Migrationsbewegungen führten die Tuberkulose vor 46000 Jahren nach Europa und Asien. Wie die Analyse der Gendrift weiter zeigt, hat die Erdbevölkerung vor etwa 10000 Jahren sprunghaft zugenommen und sich verdichtet. Diese Phase wird in der Literatur auch als Jungsteinzeit oder neolithische Revolution bezeichnet. Unter diesen Bedingungen hat sich die TB als weltweite Erkrankung endgültig etabliert.

\section{Fazit}

Genetische Rückschlüsse auf die ursprünglichen Stämme der Tuberkulose bestätigen die Vermutung, dass der Ursprung der Menschheit in Afrika liegt. Die Genmuster der Tuberkulosestämme korrespondieren mit den vorgeschichtlichen Migrationswellen der Menschheit. Dies wird als zusätzlicher wissenschaftlicher Beweis dafür gewertet, dass vor rund 10000 Jahren ein entwicklungsgeschichtlicher Sprung mit hoher Bevölkerungsverdichtung stattgefunden hat, der die Basis unserer heutigen Zivilisation bildet.

\section{Dr. Horst Gross, Berlin}

\section{Hohe Ozonwerte steigern Allergenität von Birkenpollen}

Wissenschaftler des Helmholtz Zentrums München haben in Kooperation mit ihren Kollegen von der Technischen Universität München herausgefunden, dass Ozon ein zentraler Faktor im Zusammenhang zwischen Klimawandel, Verstädterung und der Zunahme von allergischen Erkrankungen und Symptomen darstellt. Die Ergebnisse der Untersuchungen, die Ende 2013 in PLOS ONE publiziert wurden, lassen eindeutig einen Anstieg allergischer Erkrankungen erwarten.

Die Forscher untersuchten Birkenpollen, die von unterschiedlich ozonbelasteten Regionen in und um München stammten. Pricktests bestätigten eindeutig die unterschiedlichen Eigenschaften dieser Pollen. „Wir haben herausgefunden, dass Pollen unter hohen Ozonbelastungen heftigere Reaktionen bei Hauttestungen zeigten als Pollen mit anderen Standortbedingungen“, sagt Prof. Carsten Schmidt-Weber, München. „Die höhere Konzentration der Allergene in hoch ozonbelasteten Gebieten passt zu den verstärkten, entzündlichen Reaktionen, die diese Pollen hervorrufen“, erklärt Prof. Claudia Traidl-Hoffmann, München. „Ozon ist also ein prominenter Umweltfaktor mit einem großen Einfluss auf die Allergenität von Birkenpollen.“

Das Ergebnis der Forschung lässt erwarten, dass in den nächsten Jahrzehnten im Zuge des Klimawandels und der Folge zunehmender Verstädterung die Ozonkonzentrationen ansteigen und damit die Bevölkerung häufiger unter Pollenallergien leiden wird. „Ozon ist ein äußerst wichtiger Faktor, der zu einer Zunahme von Birkenpollenallergien führen wird. Es ist davon auszugehen, dass sich mit der Erhöhung der Temperatur und damit des Ozongehalts der Luft auch die Symptome von Pollenallergien vervielfachen“, erklärt Traidl-Hoffmann. „Allergiker werden deswegen nicht nur durch längere Blühphasen mit mehr Pollen, sondern auch durch potentere Pollen belastet.“

Nach einer Mitteilung des Helmholtz Zentrums München 